\title{
Genetics of Atopic Dermatitis: From DNA Sequence to Clinical Relevance
}

\author{
Mari Løset ${ }^{a, b}$ Sara J. Brown ${ }^{c, d}$ Marit Saunes ${ }^{b, e}$ Kristian Hveem ${ }^{a, f}$ \\ ${ }^{a}$ K.G. Jebsen Center for Genetic Epidemiology, Department of Public Health and Nursing, NTNU, Norwegian \\ University of Science and Technology, Trondheim, Norway; ${ }^{b}$ Department of Dermatology, St. Olavs Hospital,

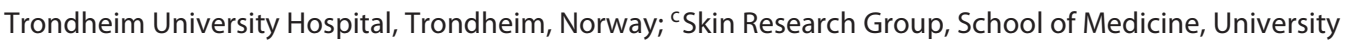

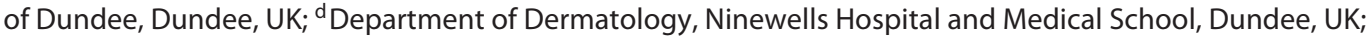 \\ e Department of Cancer Research and Molecular Medicine, NTNU, Norwegian University of Science and Technology, \\ Trondheim, Norway; ${ }^{f}$ HUNT Research Centre, Department of Public Health and Nursing, NTNU, Norwegian \\ University of Science and Technology, Trondheim, Norway
}

\section{Keywords}

Atopic dermatitis - Atopic eczema - Genetics · Genetic association - Genome-wide association studies .

Phenome-wide association studies · Sequencing ·

Mendelian randomization

\begin{abstract}
Atopic dermatitis (AD) is a complex disease that is thought to be triggered by environmental factors in genetically susceptible individuals. Twin studies have estimated the heritability of AD to be approximately $75 \%$, with the null (loss-offunction) mutations of the gene encoding filaggrin (FLG) (chromosome 1q21.3) as the strongest known genetic risk factor. The discovery of the filaggrin gene was important in the emerging model for AD pathogenesis, combining skin barrier function with adaptive and innate immunity. Assisted by the recent development of large-scale high-throughput genomics, more than 30 genetic loci have been linked to AD across different populations. Identification of these loci, together with functional studies, has already provided new insights into disease biology and identified novel drug targets. Further, these susceptibility loci are laying the groundwork for phenome-wide association studies to test their multiple
\end{abstract}

(c) 2019 S. Karger AG, Basel phenotype relationships and application of Mendelian randomization to investigate causal relationships. Despite many known genes, a majority of the genetic risk for $A D$ is yet unexplored. Therefore, studies investigating refined phenotype groups, low-frequency and rare genetic variation, gene-gene and/or gene-environment interactions, epigenetic mechanisms and data from multi-omics technologies are warranted. In this review, we describe genetic discoveries for $A D$, including results from candidate gene studies, studies of AD-like genetic diseases, genome-wide association studies and genetic sequencing studies. We explain how some of these genetic discoveries have unraveled new mechanistic insights into the pathogenesis of $A D$ and exemplify how personal genetic data could be used for preventive strategies and a tailored treatment regimen (i.e., precision medicine).

(c) 2019 S. Karger AG, Basel

\section{Introduction}

Atopic dermatitis (AD, synonymous with "atopic eczema") is a chronic inflammatory skin disease with a lifetime prevalence of $15-20 \%$ in developed countries $[1,2]$. AD
KARGER

E-Mail karger@karger.com

www.karger.com/drm
Mari Løset, $\mathrm{MD}, \mathrm{PhD}$

K.G. Jebsen Center for Genetic Epidemiology, Department of Public Health and Nursing, NTNU, Norwegian University of Science and Technology

Postboks 8905, NO-7491 Trondheim (Norway)

E-Mail mari.loset@ntnu.no 
usually begins in early childhood, and in $70 \%$ of cases the disease manifests itself before 5 years of age [3]. The disease is characterized by dry skin, pruritus, and eczematous lesions, which can lead to intense itch, sleep deprivation, and diminished self-esteem [2]. The disease shows large clinical heterogeneity related to age, ethnicity, and disease severity $[4,5]$. Consequently, topical and systemic treatments are tailored to clinical need with the aim to manage symptoms and obtain long-term disease control [6, 7].

$\mathrm{AD}$ is associated with atopic (asthma, allergic rhinitis, food allergies) and nonatopic (e.g., inflammatory diseases, psychological disturbances) comorbidities $[8,9]$. For the atopic comorbidities, the association is strong, and family and genetic studies indicate a shared genetic susceptibility [10-12]. For the nonatopic comorbidities, the association is less clear, and underlying mechanisms are likely to be complex and involve common genetic susceptibilities, systemic inflammation, environmental exposures, medication, and lifestyle factors [8].

A family history of atopic disease is the strongest known risk factor for $\mathrm{AD}$ [13]. This observation formed the rationale for genetic investigations to identify novel mechanisms leading to $\mathrm{AD}$ and related comorbidities. The search for new genetic risk factors is motivated by already highly successful discoveries in the field and in particular by the identification of the filaggrin gene (FLG) in 2006 [14]. This completely changed the understanding of atopic diseases; from a purely immunological view, the initial pathogenesis is now known to include defects of the epidermal barrier, immunological dysregulation and triggering factors [2], and this has had implications for treatment strategies $[15,16]$.

Substantial advances in the field of genetic epidemiology over the past two decades are laying the framework for new gene discoveries for complex diseases. One of the groundbreaking events in the field was when the Human Genome Project published the full human genome in $2004[17,18]$. Together with large population-based sample sizes, the innovation of cost-effective microarray technology and new analytical tools, opened for large-scale high-throughput genomics. The field has moved from candidate gene and family-based studies to large population-scale whole-genome genotyping including $>1$ million people and $>20$ million genetic variants [19].

In this review, we summarize some of the known genetic associations of $\mathrm{AD}$ in relevance to the pathogenesis of the disease and illustrate how these associations can be used for phenome-wide association studies to test their multiple phenotype relationships and application of Mendelian randomization (MR) to investigate causal re-

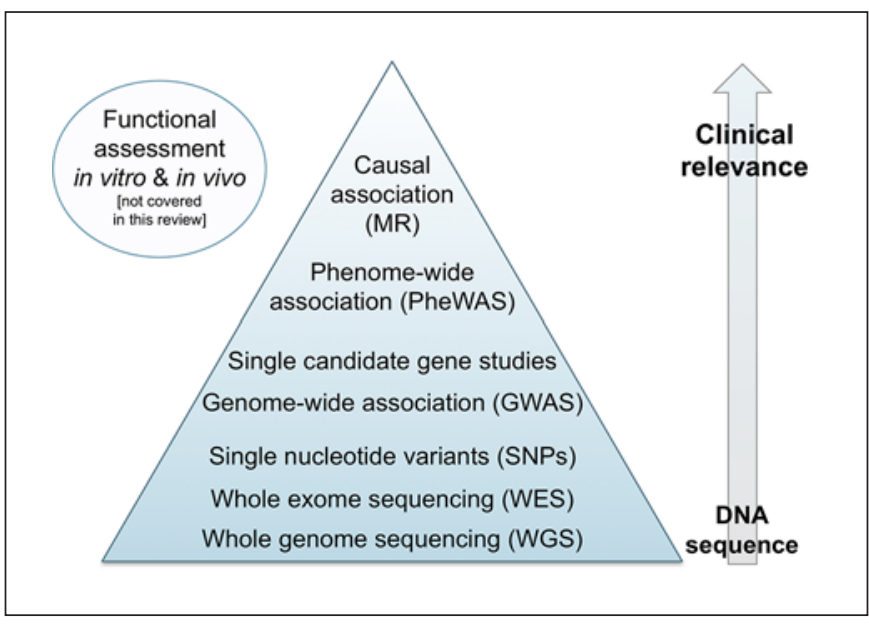

Fig. 1. Some of the investigative strategies that can be used to increase the knowledge of molecular mechanisms involved in a phenotypic trait. Candidate gene studies are performed on genes that are suggested to be involved in the genesis of a phenotypic trait because of their theoretical role in the trait being investigated. Genome-wide studies include genome-wide association studies (GWAS) and genetic sequencing studies. GWAS are mainly conducted in large population-based samples using genotype arrays that are designed to measure genetic variation at common variants. Genetic sequencing studies include both whole-exome sequencing (WES) and whole-genome sequencing (WGS). WES targets all of the protein-coding regions of the genome, and WGS targets the complete genome sequence. The results of candidate gene and genome-wide studies can be used in phenome-wide association studies (PheWAS) that analyze many phenotypic traits for association with a single genetic variant to test multiple phenotype relationships, and further, they could be used as instrumental variables in Mendelian randomization (MR) studies for the investigation of causal relationships.

lationships. An overview of some of the investigative strategies included in this paper is given in Figure 1. We focus primarily on research involving genetic studies and encourage the readers to study results from additional omics-data, i.e. epigenomics, transcriptomics, proteomics, and metabolomics in other publications [20-25]. Finally, we discuss future directions in the field including the potential avenues of precision medicine.

\section{Establishing a Genetic Component and Gene Discovery}

The heritability (the proportion of phenotypic variance due to genetic variance) of a disease or trait is usually estimated by observing patterns of inheritance among 
individuals either in family and twin studies or in population-based studies [26]. The first descriptions of familial aggregation of atopic skin disease goes 2,000 years back, when the Roman historian Suetonius described that Emperor Augustus (63 $\mathrm{BC}$ to $14 \mathrm{AD}$ ) had dry, itchy patches on his skin and suffered from a seasonal respiratory disorder, and both his grandson and his great grandnephew suffered from symptoms of atopy [27]. More recent family studies have indicated a three- to fivefold increased risk for a person to develop $\mathrm{AD}$ if one or both parents have a history of $\mathrm{AD}[13,28]$. Twin studies of $\mathrm{AD}$ show that monozygotic twins have an approximately three times higher concordance rate than dizygotic twins [29]. A systematic review which included information from 35,155 twin pairs estimated the heritability of $\mathrm{AD}$ to be approximately $75 \%$ [29], which is considered to be a high heritability for a complex trait.

\section{Candidate Gene Studies}

Filaggrin

By far the strongest known genetic risk factor for $\mathrm{AD}$ is null (loss-of-function) mutations of the gene encoding $F L G$, a gene located in the epidermal differentiation complex on chromosome 1q21.3 [14, 30, 31]. Filaggrin has multiple interrelated functions contributing to skin barrier development and maintenance [30], and null mutations of FLG lead to truncation of profilaggrin and loss of filaggrin expression. Individuals with filaggrin deficiency experience an increased skin permeability, facilitating the effect of environmental allergens, irritants and microbes, and initiation of an inflammatory cascade. The link between FLG and AD was identified in 2006 when studying ichthyosis vulgaris [32], a genetic disease that displays AD-like characteristics. FLG was further associated with $\mathrm{AD}$ [14], a discovery that changed the view of the pathogenesis of AD. The focus shifted from an immunological view including imbalance of $\mathrm{T}$ cells to inclusion of a functional disruption of the epidermal barrier as the primary pathogenic process [33]. About 10\% of the European and Japanese ancestry population carry a null mutation within FLG exon 3 and they have mild ichthyosis and a threefold increased risk of AD compared with the general population $[2,30]$. This demonstrates a strong effect for a single gene in the context of a complex disease [34].

\section{Other Genes}

Based on their theoretical role in disease pathogenesis, a large number of candidate gene studies have been performed to elucidate the genetic background of $\mathrm{AD}[20,35$, 36]. This includes genes involved in epidermal differen- tiation, skin immunity, or systemic immunity [36]. Highlighted genes identified by candidate gene studies comprise the genes encoding interleukin (IL)-4, the IL-4 receptor, and IL-13 $[37,38]$, all lying in the Th2 cytokine cluster on chromosome 5q31.1. This locus is also robustly associated with $\mathrm{AD}$ in genome-wide association studies (GWAS) (see below).

\section{Studies of AD-Like Genetic Diseases}

Detailed studies of genetic diseases that display $\mathrm{AD}$ like characteristics have provided insight into monogenic drivers of disease pathogenesis [39]. These studies have focused on diseases characterized by skin barrier dysfunction (e.g., ichthyosis vulgaris, generalized peeling skin, Netherton syndrome), multisystem atopic inflammation (e.g., severe dermatitis, multiple allergies and metabolic wasting, SAM) and immunodeficiency with skin manifestations resembling $\mathrm{AD}$ (e.g. hyper-IgE, Omenn syndrome) [39]. To illustrate, the syndrome of SAM is a rare (prevalence $<1 / 1$ million) genetic disease that shows AD-like manifestations including congenital erythroderma, superficial skin erosions, fine scales and palmoplantar keratoderma, food allergies and increased IgE levels. Whole-exome sequencing revealed homozygous loss-of-function mutations in DSG1 (encoding desmoglein) [40] and heterozygous mutation in DSP (encoding desmoplakin) $[40,41]$ that segregated with disease. The genetic mutations of SAM lead to compromised barrier function, and DSG1 deficiency was associated with increased expression of genes encoding allergy-related cytokines. This informed the knowledge of epidermal barrier dysfunctions leading to local and systemic atopic inflammation [40, 41].

\section{Genome-Wide Association Studies}

GWAS is an experimental design used to test the association between thousands, and even millions, of genetic variants with a disease outcome or trait [42]. The primary aim of these studies is to increase the understanding of the genetic risk of disease and to provide a foundation for functional and mechanistic follow-up studies that will enable more effective detection, prevention, and treatment. GWAS have proven successful at mapping areas on the human chromosomes (loci) that influence AD [43-50], and since the first published study on $\mathrm{AD}$ in 2009 [43], >30 genetic loci have been identified, where the majority show a slight to moderate risk for disease development [47]. An overview of these loci, their likely candidate genes, and known or proposed functions have recently been published $[2,4]$, including a review 


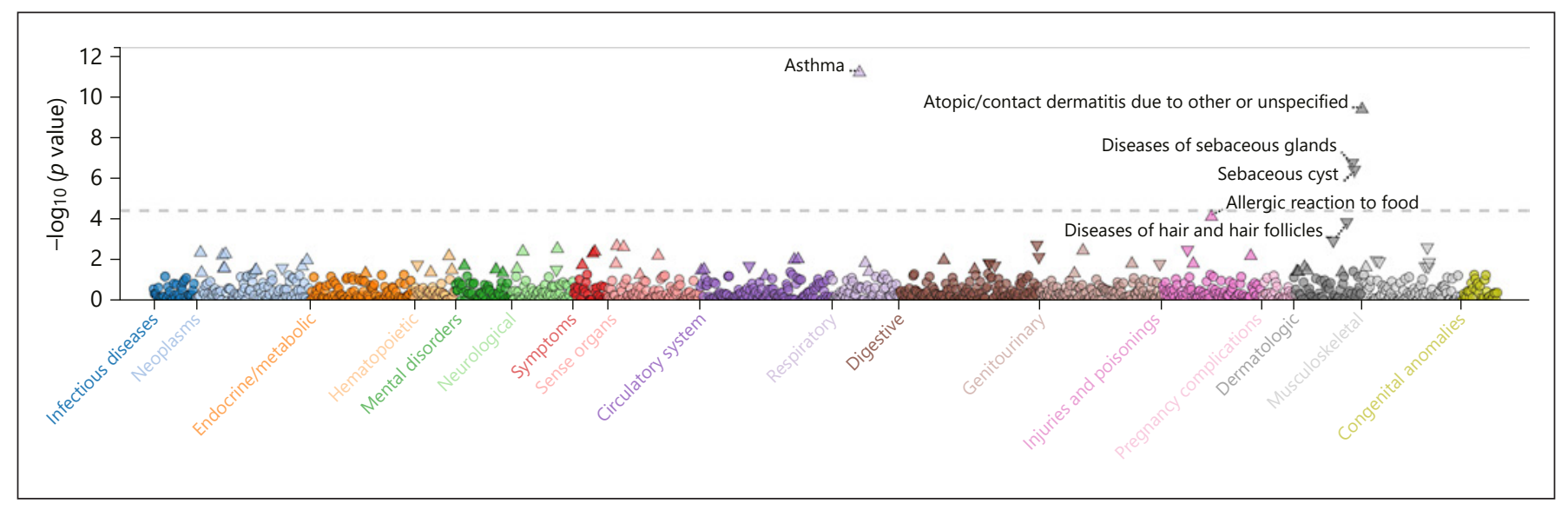

Fig. 2. Phenome-wide association study plot of the lead variant (rs61816761) in FLG in the UK Biobank. The variant is associated with dermatitis $\left(p\right.$ value $\left.3.2 \times 10^{-10}\right)$ in 2,110 cases and 40,4817 controls. As expected, the variant is also associated with asthma $\left(p\right.$ value $5 \times 10^{-12}$ ) and nominally associated with allergic reaction to food $\left(p\right.$ value $\left.5.8 \times 10^{-5}\right)$. The genetic variant is further associated with diseases of sebaceous glands $(p$ value $2.1 \times$ $10^{-7}$ ) and sebaceous cysts $\left(p\right.$ value $\left.4.6 \times 10^{-7}\right)$.

focusing on associations in diverse, non-European ethnic groups [4]. The first GWAS on AD was performed on a German population of 939 individuals with AD and 975 controls as well as on 270 complete nuclear families with two affected siblings, and their replication population included 2,637 cases and 3,957 controls [43]. They detected the FLG locus and identified a novel susceptibility locus on chromosome 11q13.5 (top hit rs7927894), an intergenic region of unknown function. The study found that approximately $13 \%$ of individuals with European ancestry are homozygous for rs7927894, and that their risk to develop AD is 1.47 times that of noncarriers [43]. Subsequent GWAS replicated the FLG and chromosome 11q13.5 loci across different populations and added several new loci associated with AD risk. The majority of GWAS loci identified are involved in skin barrier development and immunological dysfunctions, in particular in innate immune signaling and T-cell activation and differentiation [2]. Even though most genetic variants that confer AD susceptibility have relatively small effect sizes, they have provided important insights into the biology of the disease and subsequently supported the genetic basis for established drugs [47] and identified potential drug targets for allergic diseases [10].

Since the first GWAS in 2009, large-scale collaborations have made progressively larger sample sizes available, and the process of predicting genotypes through imputation, a method to estimate the genotype at genetic markers that are not directly genotyped, is commonly used. In 2015, the EAGLE consortium meta-analyzed $>15$ million genetic variants in 21,399 cases and 95,464 controls [47]. Their replication phase included 32,059 cases and 228,628 controls. The study included populations of European, African, Japanese and Latino ancestry, and identified 10 new risk loci, increasing the total number of known $\mathrm{AD}$ risk loci to 31 [47]. Of the identified loci, several are common for other atopic conditions (asthma, allergic sensitization, selfreported allergy), supporting common atopic mechanisms [47]. This was further investigated by Ferreira et al. [10], where genetic risk factors were identified between asthma, hay fever, and eczema. They identified 136 independent risk variants, of which 73 were novel; disease-specific effects were detected for only 6 variants, confirming a shared genetic risk across atopic phenotypes. The risk variants were shown to dysregulate the expression of immune-related genes [10]. These results were further explored using gene-based tests, and additional 11 risk loci for allergic diseases were discovered [11].

\section{Phenome-Wide Association Studies}

While GWAS analyze thousands to millions of genetic variants per phenotypic trait, phenome-wide association studies invert this study design to analyze many phenotypic traits for their association with a single genetic variant. The goal of this study design is to elucidate genetic variants with multiple phenotype relationships (e.g., comorbidities and pleiotropy) [51]. The method has also successfully been used for drug discovery and repositioning, prediction of adverse drug events and selection of indications for clinical trials $[52,53]$. Figure 2 shows the 
results of a phenome-wide GWAS analysis on the UK Biobank PheWeb (http://pheweb.sph.umich.edu:5003) based on GWAS of 28 million variants across 1,403 ICDderived traits identified in 408,961 individuals [54] for the lead variant (rs61816761) within the FLG gene. As expected and due to known atopic comorbidities, we find that this genetic variant is associated with asthma, dermatitis, and allergic reaction to food (the latter only nominally significant). This variant is also associated with diseases of sebaceous glands, a result that is suggestive of pleiotropy or shared disease mechanisms.

\section{Genetic Sequencing Studies}

To date, GWAS has mainly been conducted using genotype arrays that are designed to measure genetic variation at common variants. Imputation allows for estimation of genotypes of rare variants not included on genotyping arrays, but this is dependent on the size and quality of the imputation reference panel. By their nature, rare variants are so infrequently seen that those detected often do not reach statistical significance in GWAS, even in large sample sizes. Due to this, rare genetic variants (population minor allele frequency typically $<1 \%$ ) have not yet been investigated to the same extent as common variants, even though they may have from moderate to high effects on complex traits $[55,56]$. Facilitated by the steady drop in sequencing costs, large-scale studies of rare variants are now possible through both whole-exome sequencing (WES) and whole-genome sequencing (WGS). WES targets all of the protein-coding regions of the genome (known as the exome) and has proven to be a useful tool to investigate rare diseases and in the search for rare variants causing polygenic diseases [55]. For $A D$, the WES studies performed to date have had relatively small sample sizes [57-62]. The first published study included 22 Ethiopian individuals with ichthyosis vulgaris and $\mathrm{AD}$, and identified several rare variants suggesting a heterogenous disease pathogenesis [57]. Recently, 43 probands of 42 Bangladeshi families with severe AD were investigated using WES in combination with rare variant enrichment analysis [60]. This identified a rare putative loss-of-function allele in FLG as the major component of disease susceptibility, in addition to potential novel risk genes within chromosome 1 (TCHHL1, ADCY10, MTF1, MAST2), 6 (SCAND3), 9 (ORM2), 10 (MCM10), 11 (PHLDB1, $P A N X 3$ ) and 12 (CUX2) [60]. Further, WES showed that dominant negative loss-of-function mutations in CARD14 were associated with severe AD [62]. Upregulation of the same gene leads to psoriasis $[63,64]$, in line with previously identified shared loci with opposing

Genetics of Atopic Dermatitis mechanisms for psoriasis and AD [48]. Larger and population-based WES and WGS to systematically investigate less frequent genetic variations and the association with disease are ongoing in several cohorts [65]. In October 2018, it was announced that the United Kingdom's National Health Services are currently planning to sequence the genomes of one million people, and in the next 5 years, they are planning to sequence 5 million genomes [66].

\section{Investigating Causal Relationships Using MR}

Causal relationships can be investigated with MR, a method that uses genetic variants to make causal inference of nongenetic environmental exposures [67]. These studies leverage Mendel's second law that the inheritance of one trait is independent of other traits. When satisfied, genetic variants can be used as instrumental variables to estimate causal effects. Additional, germline genetic variants are fixed at conception, and this happens prior to the onset of disease, therefore these analyses are less susceptible to confounding and reverse causality $[67,68]$. Through GWAS, the increasing identification of genetic determinants of modifiable exposures and phenotypes has provided a valuable source of data to use for MR analyses. Examples of MR used in dermatology include investigations of direction(s) of causality for psoriasis with higher C-reactive protein levels (supports a noncausal relationship) [69] and higher body mass index (BMI) (supports a causal relationship) $[70,71]$. For $\mathrm{AD}$, the relationship to lower vitamin $\mathrm{D}$ levels and $\mathrm{AD}$ has been tested, and evidence indicates a noncausal relationship [72]. Observational epidemiological studies have reported an association of high $\mathrm{BMI}$ with risk of $\mathrm{AD}$ [73]. If a high $\mathrm{BMI}$ is a causal risk factor for $\mathrm{AD}$, this may have implications for treatment and prevention strategies because the $\mathrm{BMI}$ is potentially modifiable. An ongoing MR study is examining this potential causal relationship (unpubl. data). The study population includes $>400,000$ individuals from the UK Biobank and the Nord-Trøndelag Health Study (the HUNT Study) and genetic variants identified by GWAS to be associated with BMI and AD as instrumental variables.

\section{Translation into Clinical Relevance}

The results of MR studies as described above may have implications for treatment and prevention strategies for AD. Further, genetic information leading to focus on 


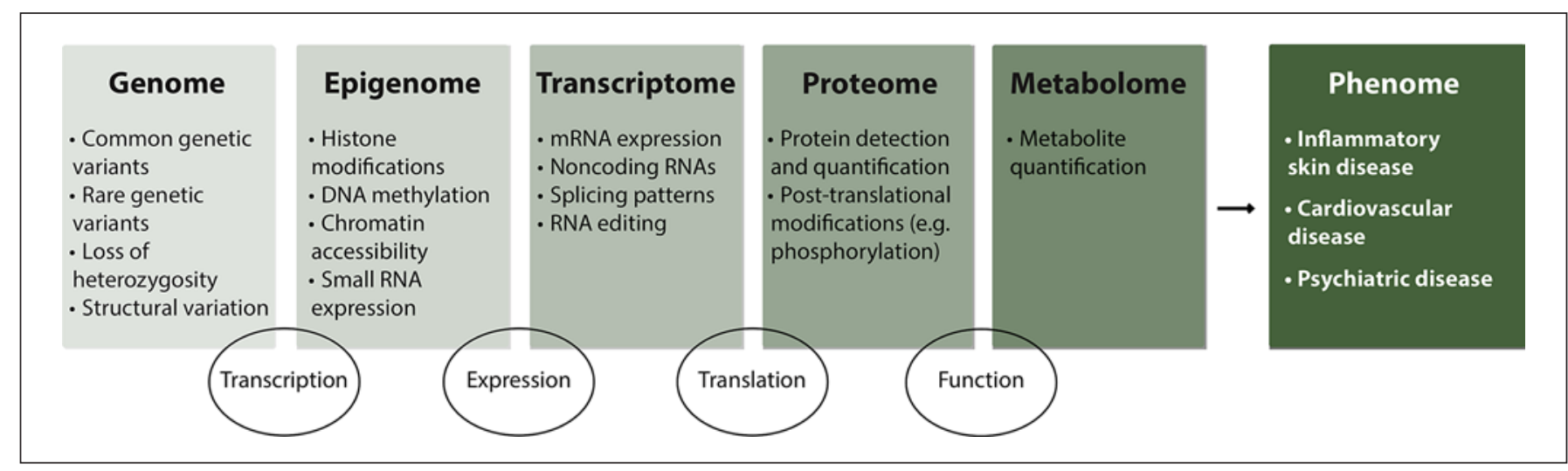

Fig. 3. Technological advances have made efficient integration of multi-omics possible. This includes combined studies of the genome, epigenome, transcriptome, proteome and metabolome, and the observed phenome. Adapted from Ritchie et al. [86].

skin barrier dysfunction as the key precursor to increased skin permeability, inflammation, and percutaneous allergic sensitization [5] have given rise to the development of novel treatment strategies that target the skin barrier or cutaneous inflammation $[15,16]$. However, despite an increasing knowledge about disease mechanisms, there is still a very limited number of systemic immunomodulating biologics that have been approved for the treatment of moderate-to-severe AD [74]. One exception is dupilumab, a fully human monoclonal antibody targeting the IL-4 and IL-13 pathway [75]. Dupilumab was the first biologic to receive US Food and Drug Administration and European Medicines Agency approval for the treatment of adults with moderate-to-severe AD. Clinical trials have shown that dupilumab significantly improved clinical outcomes and quality of life in adults with moderate-to-severe $\mathrm{AD}$ [76]. Many other new treatments are under development with several biologics and small molecule antagonists in phase II and phase III trials [2].

AD shows large clinical heterogeneity, and a recent study divided AD into 6 subphenotypes, which showed different risk profiles and diverse prognoses [77]. With the increasing knowledge of genetic associations with $\mathrm{AD}$ in combination with the steady drop in genotyping costs, one can foresee a future where health care providers could have access to the patient's genetic data [78]. Personal genetics could help stratifying individuals into $\mathrm{AD}$ disease subsets, and direct medical interventions by making predictions about disease risk, tailoring the right therapeutic strategy and prevention programs for the right person at the right time ("the promise of precision medicine"). This could again reduce the risk of adverse events and the costs related to treatments. For AD, the genetic risk could be ascertained from birth and thereby identify individuals at risk for atopic diseases. Such knowledge could allow early interventions to prevent disease, e.g. using daily emollients to enhance a defective skin barrier $[79,80]$. It has been suggested that early preventive treatments of $\mathrm{AD}$ could reduce the development of associated comorbidities, such as asthma [15]. To calculate a polygenic risk score per individual for AD could be a method of identifying individuals for preventive measures. A polygenic risk score adds together the contributions of all the small genetic effects as estimated through GWAS and provides a continuous and quantitative measure of genetic risk [81]. Polygenic risk scores could create one of the most powerful genetic diagnostics to date, such as demonstrated for e.g. coronary artery disease and atrial fibrillation [82]. To date, there is no routine in the integration of genetic testing in the clinics for inflammatory skin disease. However, work to predict individual treatment responses in dermatology is ongoing through e.g. the Psoriasis Stratification to Optimize Relevant Therapy (PSORT) consortium [83].

\section{Mind the Gap}

Despite the success of association analyses among complex diseases [42], there is still a significant proportion of genetic risk that has not yet been explained for most traits. This phenomenon has been called the "missing heritability" of complex diseases $[84,85]$. For $\mathrm{AD}$, the 
identified GWAS loci explain approximately $15 \%$ of the variance in liability [47], which is in line with other highly heritable complex traits. There are many factors that could explain this still "missing heritability" 10 years after the first large-scale GWAS. This includes effects due to marked heterogeneity of $\mathrm{AD}$, the cumulative effects of multiple genetic variations, rare genetic variations, structural variations of the genome such as copy number variants, the existence of gene-gene and/or gene-environment interactions, and heritable epigenetic mechanisms [26]. The present times are highly promising for investigating more of these effects.

Technological advances have opened new possibilities for analyzing multi-omics data [86]. The likelihood of identifying a true gene or pathway increases if data from multiple levels of biological data support the same association [86]. By multistaged and metadimensional analyses of multi-omics one could identify effective models that predict disease status, discover biomarkers, and lead to an increased understanding of the role of genetics and genomics in complex traits [86] and in $\mathrm{AD}$ in particular $[25,39]$. Multiple levels of biological data are illustrated in Figure 3 (adapted from Ritchie et al. [86]), and includes data from the genome, epigenome, transcriptome, proteome, and metabolome.

\section{Conclusions}

Over the last decade there has been a paradigm shift in our understanding of the mechanisms causing AD. Moving away from a purely immunological view, genetic studies have played a major role in understanding defects of the epidermal barrier. In the future we hope for similar mechanistic breakthroughs led by gene discoveries, that these discoveries will continue to inform drug development efforts and safety measures, and that personal genetic information may be included in routine clinical care to aid prevention and treatment of $\mathrm{AD}$. The groundwork for these implementations is the growing large-scale high-throughput genomics, including both WES and WGS, made possible by population-based biobanks in combination with information from electronic health records, and methods developed to handle these complex health data with the necessary computational and statistical expertise [65]. Together, such activities will increase the interpretation and functional validation, and future studies will most likely take gene-gene and gene-environmental effects into larger account and include multiomics technologies.

Genetics of Atopic Dermatitis

\section{Key Message}

Genetic discoveries have added significantly to the biological understanding of atopic dermatitis. In this review, we describe some of the methods used for this purpose, their findings, and future directions in the field.

\section{Acknowledgments}

The authors would like to thank Janne Tellefsen for help with the design of Figure 3, Ben M. Brumpton for commenting on the paper and revising the section on Mendelian randomization, and Brooke N. Wolford for insights on genetic sequencing studies and constructive criticism of the paper. We acknowledge researchers whose work we could not cite because of space constraints.

\section{Statement of Ethics}

The authors have no ethical conflicts to disclose.

\section{Disclosure Statement}

M.L., S.J.B., M.S., and K.H. declare no relevant conflicts of interest.

\section{Funding Sources}

M.L. and K.H. work in a research unit funded by Stiftelsen Kristian Gerhard Jebsen; Faculty of Medicine and Health Sciences, NTNU; the Liaison Committee for education, research and innovation in Central Norway; and the Joint Research Committee between St. Olavs Hospital and the Faculty of Medicine and Health Sciences, NTNU. M.L. was supported by a research grant from the Liaison Committee for education, research and innovation in Central Norway. S.J.B. holds a Wellcome Trust Senior Research Fellowship in Clinical Science (106865/Z/15/Z).

\section{Author Contributions} thors.

This paper was planned and written in collaboration by the au- 


\section{References}

1 Deckers IA, McLean S, Linssen S, Mommers $\mathrm{M}$, van Schayck CP, Sheikh A. Investigating international time trends in the incidence and prevalence of atopic eczema 1990-2010: a systematic review of epidemiological studies. PLoS One. 2012;7(7):e39803.

2 Weidinger S, Beck LA, Bieber T, Kabashima $\mathrm{K}$, Irvine AD. Atopic dermatitis. Nat Rev Dis Primers. 2018 Jun;4(1):1.

3 Williams HC. Clinical practice. Atopic dermatitis. N Engl J Med. 2005 Jun;352(22): 2314-24.

4 Kaufman BP, Guttman-Yassky E, Alexis AF. Atopic dermatitis in diverse racial and ethnic groups-Variations in epidemiology, genetics, clinical presentation and treatment. Exp Dermatol. 2018 Apr;27(4):340-57.

5 Weidinger S, Novak N. Atopic dermatitis. Lancet. 2016 Mar;387(10023):1109-22.

6 Nygaard U, Deleuran M, Vestergaard C. Emerging Treatment Options in Atopic Dermatitis: topical Therapies. Dermatology. 2017;233(5):333-43.

7 Nygaard U, Vestergaard C, Deleuran M. Emerging Treatment Options in Atopic Dermatitis: systemic Therapies. Dermatology. 2017;233(5):344-57.

8 Andersen YM, Egeberg A, Skov L, Thyssen JP. Comorbidities of atopic dermatitis: beyond rhinitis and asthma. Curr Dermatol Rep. 2017;6(1):35-41

9 Dalgard FJ, Gieler U, Tomas-Aragones L, Lien L, Poot F, Jemec GB, et al. The psychological burden of skin diseases: a cross-sectional multicenter study among dermatological out-patients in 13 European countries. J Invest Dermatol. 2015 Apr;135(4):984-91.

10 Ferreira MA, Vonk JM, Baurecht H, Marenholz I, Tian C, Hoffman JD, et al.; 23andMe Research Team; AAGC collaborators; BIOS consortium; LifeLines Cohort Study. Shared genetic origin of asthma, hay fever and eczema elucidates allergic disease biology. Nat Genet. 2017 Dec;49(12):1752-7.

11 Ferreira MA, Vonk JM, Baurecht H, Marenholz I, Tian C, Hoffman JD, et al. Eleven loci with new reproducible genetic associations with allergic disease risk. J Allergy Clin Immunol. 2019 Feb;143(2):691-9.

12 Pinart M, Benet M, Annesi-Maesano I, von Berg A, Berdel D, Carlsen KC, et al. Comorbidity of eczema, rhinitis, and asthma in IgEsensitised and non-IgE-sensitised children in MeDALL: a population-based cohort study. Lancet Respir Med. 2014 Feb;2(2):131-40.

13 Apfelbacher CJ, Diepgen TL, Schmitt J. Determinants of eczema: population-based cross-sectional study in Germany. Allergy. 2011 Feb;66(2):206-13.

14 Palmer CN, Irvine AD, Terron-Kwiatkowski A, Zhao Y, Liao H, Lee SP, et al. Common loss-of-function variants of the epidermal barrier protein filaggrin are a major predisposing factor for atopic dermatitis. Nat Genet. 2006 Apr;38(4):441-6.
15 Tsakok T, Woolf R, Smith CH, Weidinger S, Flohr C. Atopic dermatitis: the skin barrier and beyond. Br J Dermatol. 2019 Mar;180(3): 464-74.

16 Elias PM, Wakefield JS. Therapeutic implications of a barrier-based pathogenesis of atopic dermatitis. Clin Rev Allergy Immunol. 2011 Dec;41(3):282-95.

17 Roberts L, Davenport RJ, Pennisi E, Marshall E. A history of the Human Genome Project. Science. 2001 Feb;291(5507): 1195.

18 McPherson JD, Marra M, Hillier L, Waterston RH, Chinwalla A, Wallis J, et al.; International Human Genome Mapping Consortium. A physical map of the human genome. Nature. 2001 Feb;409(6822):934-41.

19 Nielsen JB, Thorolfsdottir RB, Fritsche LG, Zhou W, Skov MW, Graham SE, et al. Biobank-driven genomic discovery yields new insight into atrial fibrillation biology. Nat Genet. 2018 Sep;50(9):1234-9.

20 Bin L, Leung DY. Genetic and epigenetic studies of atopic dermatitis. Allergy Asthma Clin Immunol. 2016 Oct;12(1):52.

21 Suárez-Fariñas M, Ungar B, Correa da Rosa J, Ewald DA, Rozenblit M, Gonzalez J, et al. RNA sequencing atopic dermatitis transcriptome profiling provides insights into novel disease mechanisms with potential therapeutic implications. J Allergy Clin Immunol. 2015 May;135(5):1218-27.

22 Scrivo R, Casadei L, Valerio M, Priori R, Valesini G, Manetti C. Metabolomics approach in allergic and rheumatic diseases. Curr Allergy Asthma Rep. 2014 Jun;14(6):445.

23 Ottas A, Fishman D, Okas TL, Püssa T, Toomik P, Märtson A, et al. Blood serum metabolome of atopic dermatitis: altered energy cycle and the markers of systemic inflammation. PLoS One. 2017 Nov;12(11):e0188580.

24 Oliva M, Renert-Yuval Y, Guttman-Yassky E. The 'omics' revolution: redefining the understanding and treatment of allergic skin diseases. Curr Opin Allergy Clin Immunol. 2016 Oct;16(5):469-76.

25 Ghosh D, Bernstein JA, Khurana Hershey GK, Rothenberg ME, Mersha TB. Leveraging multilayered "omics" data for atopic dermatitis: a road map to precision medicine. Front Immunol. 2018 Dec;9:2727.

26 Verma SS, Ritchie MD. Another Round of "Clue" to Uncover the Mystery of Complex Traits. Genes (Basel). 2018 Jan;9(2):E61.

27 Mier PD. Earliest description of the atopic syndrome? Br J Dermatol. 1975;92(3):359.

28 Wadonda-Kabondo N, Sterne JA, Golding J, Kennedy CT, Archer CB, Dunnill MG; ALSPAC Study Team. Association of parental eczema, hayfever, and asthma with atopic dermatitis in infancy: birth cohort study. Arch Dis Child. 2004 Oct;89(10):917-21.

29 Elmose C, Thomsen SF. Twin studies of atopic dermatitis: interpretations and applications in the filaggrin era. J Allergy (Cairo). 2015; 2015:902359.
30 Irvine AD, McLean WH, Leung DY. Filaggrin mutations associated with skin and allergic diseases. N Engl J Med. 2011 Oct;365(14): 1315-27.

31 Rodriguez E, Baurecht H, Herberich E, Wagenpfeil S, Brown SJ, Cordell HJ, et al. Metaanalysis of filaggrin polymorphisms in eczema and asthma: robust risk factors in atopic disease. J Allergy Clin Immunol. 2009;123(6): 1361-70. e7.

32 Smith FJ, Irvine AD, Terron-Kwiatkowski A, Sandilands A, Campbell LE, Zhao Y, et al. Loss-of-function mutations in the gene encoding filaggrin cause ichthyosis vulgaris. Nat Genet. 2006 Mar;38(3):337-42.

33 Elias PM, Steinhoff M. "Outside-to-inside" (and now back to "outside") pathogenic mechanisms in atopic dermatitis. J Invest Dermatol. 2008 May;128(5):1067-70.

34 Brown SJ. Molecular mechanisms in atopic eczema: insights gained from genetic studies. J Pathol. 2017 Jan;241(2):140-5.

35 Barnes KC. An update on the genetics of atopic dermatitis: scratching the surface in 2009. J Allergy Clin Immunol. 2010;125(1):16-29. e1-11; quiz 30-1.

36 Brown SJ, McLean WH. Eczema genetics: current state of knowledge and future goals. J Invest Dermatol. 2009 Mar;129(3):543-52.

37 He JQ, Chan-Yeung M, Becker AB, DimichWard H, Ferguson AC, Manfreda J, et al. Genetic variants of the IL13 and IL4 genes and atopic diseases in at-risk children. Genes Immun. 2003 Jul;4(5):385-9.

38 Liu X, Nickel R, Beyer K, Wahn U, Ehrlich E, Freidhoff LR, et al. An IL13 coding region variant is associated with a high total serum IgE level and atopic dermatitis in the German multicenter atopy study (MAS-90). J Allergy Clin Immunol. 2000 Jul;106(1 Pt 1):167-70.

39 Eyerich K, Brown SJ, Perez White BE, Tanaka RJ, Bissonette R, Dhar S, et al. Human and computational models of atopic dermatitis: a review and perspectives by an expert panel of the International Eczema Council. J Allergy Clin Immunol. 2019 Jan;143(1):36-45.

40 Samuelov L, Sarig O, Harmon RM, Rapaport D, Ishida-Yamamoto A, Isakov O, et al. Desmoglein 1 deficiency results in severe dermatitis, multiple allergies and metabolic wasting. Nat Genet. 2013 Oct;45(10):1244-8.

41 McAleer MA, Pohler E, Smith FJ, Wilson NJ, Cole C, MacGowan S, et al. Severe dermatitis, multiple allergies, and metabolic wasting syndrome caused by a novel mutation in the $\mathrm{N}$ terminal plakin domain of desmoplakin. J Allergy Clin Immunol. 2015 Nov;136(5):126876.

42 Visscher PM, Wray NR, Zhang Q, Sklar P, McCarthy MI, Brown MA, et al. 10 Years of GWAS Discovery: Biology, Function, and Translation. Am J Hum Genet. 2017 Jul; 101(1):5-22. 
43 Esparza-Gordillo J, Weidinger S, FölsterHolst R, Bauerfeind A, Ruschendorf F, Patone $\mathrm{G}$, et al. A common variant on chromosome $11 \mathrm{q} 13$ is associated with atopic dermatitis. Nat Genet. 2009 May;41(5):596-601.

44 Sun LD, Xiao FL, Li Y, Zhou WM, Tang HY, Tang XF, et al. Genome-wide association study identifies two new susceptibility loci for atopic dermatitis in the Chinese Han population. Nat Genet. 2011 Jun;43(7):690-4.

45 Hirota T, Takahashi A, Kubo M, Tsunoda T, Tomita K, Sakashita M, et al. Genome-wide association study identifies eight new susceptibility loci for atopic dermatitis in the Japanese population. Nat Genet. 2012 Nov;44(11): $1222-6$.

46 Weidinger S, Willis-Owen SA, Kamatani Y, Baurecht H, Morar N, Liang L, et al. A genome-wide association study of atopic dermatitis identifies loci with overlapping effects on asthma and psoriasis. Hum Mol Genet. 2013 Dec;22(23):4841-56.

47 Paternoster L, Standl M, Waage J, Baurecht $\mathrm{H}$, Hotze M, Strachan DP, et al.; Australian Asthma Genetics Consortium (AAGC). Multi-ancestry genome-wide association study of 21,000 cases and 95,000 controls identifies new risk loci for atopic dermatitis. Nat Genet. 2015 Dec;47(12):1449-56.

48 Baurecht $\mathrm{H}$, Hotze M, Brand S, Büning C, Cormican P, Corvin A, et al.; Psoriasis Association Genetics Extension. Genome-wide comparative analysis of atopic dermatitis and psoriasis gives insight into opposing genetic mechanisms. Am J Hum Genet. 2015 Jan; 96(1):104-20.

49 Schaarschmidt H, Ellinghaus D, Rodríguez E, Kretschmer A, Baurecht H, Lipinski S, et al. A genome-wide association study reveals 2 new susceptibility loci for atopic dermatitis. J Allergy Clin Immunol. 2015 Sep;136(3):802-6.

50 Kim KW, Myers RA, Lee JH, Igartua C, Lee $\mathrm{KE}$, Kim YH, et al. Genome-wide association study of recalcitrant atopic dermatitis in Korean children. J Allergy Clin Immunol. 2015 Sep;136(3):678-684.e4.

51 Bush WS, Oetjens MT, Crawford DC. Unravelling the human genome-phenome relationship using phenome-wide association studies. Nat Rev Genet. 2016 Mar;17(3):129-45.

52 Diogo D, Bastarache L, Liao KP, Graham RR, Fulton RS, Greenberg JD, et al. TYK2 proteincoding variants protect against rheumatoid arthritis and autoimmunity, with no evidence of major pleiotropic effects on non-autoimmune complex traits. PLoS One. 2015 Apr; 10(4):e0122271.

53 Rastegar-Mojarad M, Ye Z, Kolesar JM, Hebbring SJ, Lin SM. Opportunities for drug repositioning from phenome-wide association studies. Nat Biotechnol. 2015 Apr;33(4):3425 .
54 Zhou W, Nielsen JB, Fritsche LG, Dey R, Gabrielsen ME, Wolford BN, et al. Efficiently controlling for case-control imbalance and sample relatedness in large-scale genetic association studies. Nat Genet. 2018 Sep;50(9): 1335-41.

55 Rabbani B, Tekin M, Mahdieh N. The promise of whole-exome sequencing in medical genetics. J Hum Genet. 2014 Jan;59(1):5-15.

56 Turcot V, Lu Y, Highland HM, Schurmann C, Justice AE, Fine RS, et al.; CHD Exome+ Consortium; EPIC-CVD Consortium; ExomeBP Consortium; Global Lipids Genetic Consortium; GoT2D Genes Consortium; EPIC InterAct Consortium; INTERVAL Study; ReproGen Consortium; T2D-Genes Consortium; MAGIC Investigators; Understanding Society Scientific Group. Protein-altering variants associated with body mass index implicate pathways that control energy intake and expenditure in obesity. Nat Genet. 2018 Jan;50(1):26-41.

57 Taylan F, Nilsson D, Asad S, Lieden A, Wahlgren $\mathrm{CF}$, Winge $\mathrm{MC}$, et al. Whole-exome sequencing of Ethiopian patients with ichthyosis vulgaris and atopic dermatitis. J Allergy Clin Immunol. 2015;136(2):507-9. e19.

58 Heo WI, Park KY, Jin T, Lee MK, Kim M, Choi $\mathrm{EH}$, et al. Identification of novel candidate variants including COL6A6 polymorphisms in early-onset atopic dermatitis using whole-exome sequencing. BMC Med Genet. 2017 Jan;18(1):8.

59 Luo W, Xu W, Xia L, Xie D, Wang L, Guo Z et al. Family-based whole exome sequencing of atopic dermatitis complicated with cataracts. Oncotarget. 2017 Jul;8(35):59446-54.

60 Pigors M, Common JE, Wong XF, Malik S, Scott CA, Tabarra N, et al. Exome Sequencing and Rare Variant Analysis Reveals Multiple Filaggrin Mutations in Bangladeshi Families with Atopic Eczema and Additional Risk Genes. J Invest Dermatol. 2018 Dec;138(12): 2674-7.

61 Heo WI, Park KY, Lee MK, Kim JH, Moon NJ, Seo SJ. Association of CDKAL1 polymorphisms with early-onset atopic dermatitis in Koreans. Ann Dermatol. 2018 Jun;30(3):27683.

62 Peled A, Sarig O, Sun G, Samuelov L, Ma CA, Zhang Y, et al. Loss-of-function mutations in CARD14 are associated with a severe variant of atopic dermatitis. J Allergy Clin Immunol. 2019 Jan;143(1):173-181.

63 Jordan CT, Cao L, Roberson ED, Duan S, Helms CA, Nair RP, et al. Rare and common variants in CARD14, encoding an epidermal regulator of NF-kappaB, in psoriasis. Am J Hum Genet. 2012 May;90(5):796-808.

64 Jordan CT, Cao L, Roberson ED, Pierson KC, Yang CF, Joyce CE, et al. PSORS2 is due to mutations in CARD14. Am J Hum Genet. 2012 May;90(5):784-95.

65 Wolford BN, Willer CJ, Surakka I. Electronic health records: the next wave of complex disease genetics. Hum Mol Genet. 2018 May;27 R1:R14-21.
66 O'Dowd A. Conservative conference: Hancock pledges extra cash for social care and expansion of genomic medicine. BMJ. 2018 Oct; 363:k4156.

67 Lawlor DA, Harbord RM, Sterne JA, Timpson N, Davey Smith G. Mendelian randomization: using genes as instruments for making causal inferences in epidemiology. Stat Med. 2008 Apr;27(8):1133-63.

68 Smith GD, Ebrahim S. 'Mendelian randomization': can genetic epidemiology contribute to understanding environmental determinants of disease? Int J Epidemiol. $2003 \mathrm{Feb}$; 32(1):1-22.

69 Prins BP, Abbasi A, Wong A, Vaez A, Nolte I, Franceschini N, et al.; PAGE Consortium; International Stroke Genetics Consortium; Systemic Sclerosis consortium; Treat OA consortium; DIAGRAM Consortium; CARDIoGRAMplusC4D Consortium; ALS consortium; International Parkinson's Disease Genomics Consortium; Autism Spectrum Disorder Working Group of the Psychiatric Genomics Consortium; CKDGen consortium; GERAD1 Consortium; International Consortium for Blood Pressure; Schizophrenia Working Group of the Psychiatric Genomics Consortium; Inflammation Working Group of the CHARGE Consortium. Investigating the Causal Relationship of C-Reactive Protein with 32 Complex Somatic and Psychiatric Outcomes: A Large-Scale Cross-Consortium Mendelian Randomization Study. PLoS Med. 2016 Jun;13(6):e1001976.

70 Budu-Aggrey A, Brumpton B, Tyrrell J, Watkins S, Modalsli EH, Celis-Morales C, et al. Evidence of a causal relationship between body mass index and psoriasis: A mendelian randomization study. PLoS Med. 2019 Jan; 16(1):e1002739.

71 Ogawa K, Stuart PE, Tsoi LC, Suzuki K, Nair RP, Mochizuki H, et al. A Transethnic Mendelian Randomization Study Identifies Causality of Obesity on Risk of Psoriasis. J Invest Dermatol. 2018 Dec;S0022-202X(18)32915-4.

72 Manousaki D, Paternoster L, Standl M, Moffatt MF, Farrall M, Bouzigon E, et al. Vitamin D levels and susceptibility to asthma, elevated immunoglobulin E levels, and atopic dermatitis: A Mendelian randomization study. PLoS Med. 2017 May;14(5):e1002294.

73 Zhang A, Silverberg JI. Association of atopic dermatitis with being overweight and obese: a systematic review and metaanalysis. J Am Acad Dermatol. 2015 Apr;72(4):606-16.e4.

74 Brown SJ. What progress have we made in the treatment of atopic eczema? Putting the new biological therapies into a wider context. Br J Dermatol. 2017 Jul;177(1):4-6.

75 Beck LA, Thaçi D, Hamilton JD, Graham NM, Bieber T, Rocklin R, et al. Dupilumab treatment in adults with moderate-to-severe atopic dermatitis. N Engl J Med. 2014 Jul; 371(2):130-9. 
76 Gooderham MJ, Hong HC, Eshtiaghi P, Papp KA. Dupilumab: a review of its use in the treatment of atopic dermatitis. J Am Acad Dermatol. 2018;78(3s1):S28-S36.

77 Paternoster L, Savenije OE, Heron J, Evans DM, Vonk JM, Brunekreef B, et al. Identification of atopic dermatitis subgroups in children from 2 longitudinal birth cohorts. J Allergy Clin Immunol. 2018 Mar;141(3):96471.

78 Khera AV, Kathiresan S. Genetics of coronary artery disease: discovery, biology and clinical translation. Nat Rev Genet. 2017 Jun;18(6): 331-44.

79 Horimukai K, Morita K, Narita M, Kondo M, Kitazawa H, Nozaki M, et al. Application of moisturizer to neonates prevents development of atopic dermatitis. J Allergy Clin Immunol. 2014;134(4):824-30. e6.
80 Simpson EL, Chalmers JR, Hanifin JM, Thomas KS, Cork MJ, McLean WH, et al. Emollient enhancement of the skin barrier from birth offers effective atopic dermatitis prevention. J Allergy Clin Immunol. 2014 Oct;134(4):818-23.

81 Chatterjee N, Shi J, García-Closas M. Developing and evaluating polygenic risk prediction models for stratified disease prevention. Nat Rev Genet. 2016 Jul;17(7):392-406.

82 Khera AV, Chaffin M, Aragam KG, Haas ME, Roselli C, Choi SH, et al. Genome-wide polygenic scores for common diseases identify individuals with risk equivalent to monogenic mutations. Nat Genet. 2018 Sep;50(9):121924.
83 Griffiths CE, Barnes MR, Burden AD, Nestle FO, Reynolds NJ, Smith CH, et al.; On Behalf Of The Psort Consortium. Establishing an Academic-Industrial Stratified Medicine Consortium: Psoriasis Stratification to Optimize Relevant Therapy. J Invest Dermatol. 2015 Dec;135(12):2903-7.

84 Manolio TA, Collins FS, Cox NJ, Goldstein DB, Hindorff LA, Hunter DJ, et al. Finding the missing heritability of complex diseases. Nature. 2009 Oct;461(7265):747-53.

85 Maher B. Personal genomes: the case of the missing heritability. Nature. 2008 Nov; 456(7218):18-21.

86 Ritchie MD, Holzinger ER, Li R, Pendergrass SA, Kim D. Methods of integrating data to uncover genotype-phenotype interactions. Nat Rev Genet. 2015 Feb;16(2):85-97. 